\title{
Prediction of pre-eclampsia at 11-14 weeks of pregnancy using mean arterial pressure, uterine artery Doppler and pregnancy-associated plasma protein-A
}

\author{
Shinjini Narang ${ }^{1}$, Anjoo Agarwal ${ }^{1 *}$, Vinita Das ${ }^{1}$, Amita Pandey $^{1}$, Smriti Agrawal $^{1}$, Wahid Ali ${ }^{2}$
}

${ }^{1}$ Department of Obstetrics and Gynaecology, King George Medical University, Lucknow, Uttar Pradesh, India

${ }^{2}$ Department of Pathology, King George Medical University, Lucknow, Uttar Pradesh, India

Received: 05 September 2016

Accepted: 03 October 2016

\section{*Correspondence:}

Dr. Anjoo Agarwal,

E-mail: anjooa@gmail.com

Copyright: () the author(s), publisher and licensee Medip Academy. This is an open-access article distributed under the terms of the Creative Commons Attribution Non-Commercial License, which permits unrestricted non-commercial use, distribution, and reproduction in any medium, provided the original work is properly cited.

\section{ABSTRACT}

Background: The possibility of prediction of preeclampsia (PE) at 11-14 weeks of pregnancy is a fairly new concept and in recent years, studies combining various parameters at 11-14 weeks of pregnancy have been undertaken, but an algorithm with a high predictive value is yet to be developed. The objective of this study was to develop such a protocol using mean arterial pressure (MAP), uterine artery Doppler and PAPP-A (pregnancy associated plasma protein-A) at 11-14 weeks of pregnancy (individually or in combination) for prediction of preeclampsia in a developing country like India.

Methods: A prospective cohort study was done at the Department of Obstetrics and Gynecology, King George Medical University, Lucknow, Uttar Pradesh with pregnant women attending the antenatal OPD at $11-14$ weeks of gestation. A preformed questionnaire was filled for the enrolled women, MAP, blood pressure was recorded, uterine artery Doppler was done, serum sample for PAPP-A was drawn. Uterine artery Doppler pulsatility index (PI) at 11-14 weeks of pregnancy was found to be a good screening method (sensitivity-75.9\%, specificity-79.6\% at cut-off of 1.7) for prediction of preeclampsia and IUGR. The data was analyzed using SPSS (Statistical Package for Social Sciences) Version 15.0 statistical analysis software.

Results: Out of the 78 women enrolled, 29 women $(37.18 \%)$ developed complications. Uterine artery Doppler pulsatility index (PI) at 11-14 weeks of pregnancy was found to be a good screening method (sensitivity-75.9\%, specificity-79.6\%) for prediction of preeclampsia and IUGR. Presence of early diastolic notch on uterine artery Doppler was found predictive for IUGR. MAP and PAPP-A were not found to have a significant correlation with development of these complications.

Conclusions: This study concluded that uterine artery Doppler alone was a good screening method at 11-14 weeks of gestation for women at high risk of developing preeclampsia and related complications.

Keywords: PAPP-A, Preeclampsia, MAP, Uterine artery Doppler

\section{INTRODUCTION}

Pre-eclampsia (PE) is a pregnancy-specific syndrome that can affect virtually every organ system. It affects $5-10 \%$ of pregnancies worldwide and $4.6 \%$ of pregnancies in India. ${ }^{1,2}$ It forms one member of the deadly triad, along with hemorrhage and infection that contribute greatly to maternal morbidity and mortality (14\% worldwide, $29.54 \%$ in India). ${ }^{3,4}$
Studies have shown that defective trophoblastic invasion of the placenta plays a crucial role in the pathogenesis of preeclampsia. Poor placentation is associated with an imbalance of circulating vasoactive factors and, in turn, leads to maternal vascular maladaptation with associated systemic endothelial dysfunction. ${ }^{5,6}$ Placental products are released as part of the placentation process. Levels of these biochemical markers reflect the pathophysiology of defective placentation, and, as a consequence, are 
assuming an increasing role in early gestation screening tests for later pregnancy complications. These biomarkers include pregnancy-associated plasma protein-A (PAPPA), placental growth factor (PIGF), soluble FMS-like tyrosine kinase-1 (sFlt-1), soluble endoglin (sEng), activin-A, inhibin-A, a disintegrin and metalloprotease 12 (ADAM12), and placental protein 13 (PP13).

PAPP-A (pregnancy associated plasma protein A) is a protease for insulin like growth factor binding protein-4 (IGFBP- 4). A low PAPP-A level is associated with higher IGFBP-4 and lower free Insulin like growth factor (IGF). IGF is known to influence fetal growth by controlling uptake of amino acids and glucose as well as having an autocrine and paracrine role in trophoblast invasion. Maternal serum PAPP-A has been shown to be relatively low in the first trimester of pregnancies complicated by IUGR and/or pre-eclampsia. ${ }^{7}$

Uterine artery Doppler waveform analysis has the potential to predict pregnancy complications associated with uteroplacental insufficiency before the onset of clinical features. Resistance to blood flow within the uteroplacental circulation is transmitted upstream to the uterine arteries. Faulty trophoblastic invasion of the spiral arteries results in diminished placental perfusion and upstream increased uterine artery resistance, this results in an increased pulsatility index (PI) or resistance index (RI) and an early diastolic notch. ${ }^{8}$

Preeclampsia is commonly divided into early-onset (diagnosed and requiring delivery < 34 weeks' gestation) and late-onset disease (diagnosed and requiring delivery $>34$ weeks' gestation). ${ }^{9}$ Early-onset preeclampsia (also known as "placental PE") results from impaired trophoblast invasion into the spiral arteries, leading to placental ischemia and oxidative stress. Placental histology in early-onset preeclampsia or IUGR often demonstrates thrombotic changes in the villous trees. On the other hand, late-onset preeclampsia ("maternal PE") is considered to be secondary to maternal cardiovascular and metabolic predisposition for endothelial dysfunction and has similar risk factors for development of cardiac disease eg. Hypertension, obesity, impaired glucose tolerance, and dyslipidemia. The placenta in such cases may appear normal or have minimal abnormalities histopathologically. Consequently, the uterine artery doppler parameters may remain within the normal range. ${ }^{10,11}$

Intrauterine fetal growth restriction (IUGR) that develops in the absence of preeclampsia is also postulated to have its origin in defective placentation. Considering the common origins of IUGR and preeclampsia (especially early-onset PE) in defective placentation and consequent uteroplacental insufficiency, first-trimester Doppler assessment of the uterine circulation may also be predictive of development of IUGR during pregnancy.
The present study was planned to evaluate the role of these early pregnancy biomarkers and Doppler changes in prediction of late pregnancy changes. All the potential biochemical markers were not used as that would increase the cost of the screening process and it is not practical to use an expensive screening tool universally in a developing nation like India. Thus, we have restricted ourselves to using only three parameters (mean arterial pressure, uterine artery Doppler and PAPP-A). Additionally, these parameters can be combined with universal fetal aneuploidy testing which is already recommended at 11-14 weeks of pregnancy. ${ }^{12}$

\section{METHODS}

The study was conducted at the Department of Obstetrics and Gynaecology, King George Medical University, Lucknow over a period of one year from September 2014 to August 2015. The samples were analysed in the Department Of Pathology, King George Medical University, Lucknow at no extra cost to the patient. Ethical clearance was obtained from the institutional ethical committee. It was a Prospective Cohort Study. 78 Pregnant women were enrolled at 11-14 weeks of gestation after taking informed consent. They were kept under follow up and their outcomes were noted till delivery. All women attending ANC OPD whether low risk or high risk were included .The exclusion criteria was - women with essential hypertension, multifetal pregnancy and those who did not give consent. A detailed questionnaire was filled which included demographic factors, obstetric history, past and family history, blood group and body mass index.

The study was done in a tertiary care centre, thus patients with complications in present or past pregnancies are referred here. So the overall incidence of preeclampsia and IUGR is higher (30\% according to previous hospital data). The sample size was calculated for $95 \%$ significance level and power of the study 0.8 by taking $30 \%$ as the incidence of expected outcomes and the expected ratio between the two groups as $3: 1^{13}$.

After routine antenatal examination, the following parameters were recorded- Mean arterial pressure (MAP), Uterine artery Doppler PI, PAPP-A.

MAP is defined as the average arterial pressure during a single cardiac cycle. The blood pressure was taken in both arms simultaneously in seated position with arms supported at the level of the heart by 2 digital sphygmomanometers (model-HEM-7121, Omron Healthcare Co., Ltd. Japan) using a normal $(22$ to $32 \mathrm{~cm}$ ) adult cuff. After rest for 5 minutes, BP was measured and a series of recordings were made at one minute intervals until variations between consecutive readings fell within $10 \mathrm{~mm} \mathrm{Hg}$ in systolic and $6 \mathrm{~mm} \mathrm{Hg}$ in diastolic BP in both arms. Then the MAP of each arm (using the average of the last 2 stable measurements) was calculated by the formula: 
$\mathrm{MAP}=\mathrm{DBP}+1 / 3(\mathrm{SBP}-\mathrm{DBP})$

Where, DBP - diastolic BP, SBP - systolic BP.

The arm with the highest final MAP was taken for the subsequent analysis of results. ${ }^{14}$

Uterine artery Doppler was done according to the ISUOG Practice Guidelines (2013) for use of Doppler Ultrasonography in Obstetrics. ${ }^{15}$ On the machine: Toshiba Aplio XG LCD Monitor Color Doppler Ultrasound (Toshiba, Japan). After emptying the bladder, the woman was laid in supine position. Transabdominally, the crown rump length or biparietal diameter was obtained and placental localization done. A midsagittal section of the uterus was obtained and the cervical canal was identified. The probe was then moved laterally until the paracervical vascular plexus was seen, colour Doppler was turned on and the uterine artery was identified as it turned cranially to make its ascent to the uterine body. Measurements were taken at this point, before the uterine artery branched into the arcuate arteries and the same process was repeated on the contralateral side. The pulsatility index (PI) was measured bilaterally and the mean PI of the uterine arteries was calculated. The presence of early diastolic notch was noted.

Maternal serum samples were collected via venepuncture. The samples were centrifuged and the serum was stored in a deep freezer $\left(-20^{\circ} \mathrm{C}\right)$ and were assayed using PAPPA ELISA kit (DRG PAPP-A ELISA EIA-2397, DRG International, Inc. USA).

The women enrolled in the study were then kept under follow up for the subsequent development of preeclampsia, intrauterine growth restriction (IUGR).

1. Pre-eclampsia (without severe disease) - systolic blood pressure $\geq 140 \mathrm{~mm} \mathrm{Hg}$ or diastolic blood pressure $\geq 90 \mathrm{~mm} \mathrm{Hg}$ (in two recordings at least $4 \mathrm{hr}$ apart in a woman with previously normal blood pressure) with proteinuria at $>20$ weeks of gestation.

a. Early onset preeclampsia (EO-PE) - developing before 34 weeks of gestation

b. Late onset preeclampsia (LO - PE) - developing after 34 weeks of gestation (9)

2. Preeclampsia (with severe disease) - blood pressure greater than or equal to $160 \mathrm{~mm} \mathrm{Hg}$ systolic or greater than or equal to $110 \mathrm{~mm} \mathrm{Hg}$ diastolic, or BP $\geq 140 / 90 \mathrm{~mm} \mathrm{Hg}$ with one or more of the following

a. thrombocytopenia- platelet count less than $100,000 /$ microliter

b. renal insufficiency

i. serum creatinine concentration greater than $1.1 \mathrm{mg} / \mathrm{dl}$ ii. or doubling of serum creatinine concentration in the absence of other renal disease

c. impaired liver function- elevated blood concentrations of liver transaminase to twice normal concentrations (SGOT/SGPT $\geq 80 \mathrm{IU} / \mathrm{L}$ )

d. pulmonary edema

e. cerebral or visual symptoms

3. Intrauterine growth restriction (IUGR)- weight of fetus below the10th percentile for their gestational age (calculated by using global reference for fetalweight and birth weight percentiles derived from the Hadlocks formula). ${ }^{17,18}$

4. Abruptio placentae: Premature separation of a normally implanted placenta

The data was analysed using SPSS (Statistical Package for Social Sciences) Version 15.0 statistical analysis software (USA). For the categorical data in the study, relative risk was calculated and chi square test was applied. For the MAP, Mean PI and PAPP-A, mean \pm SD was calculated and these were analysed by student $t$ test and receiver operating curve (ROC) was constructed.

\section{RESULTS}

Out of the 78 women enrolled, 29 women (37.18\%) developed one or more complications and the other 49 $(62.82 \%)$ had an uncomplicated pregnancy. Table 1 shows the distribution of women according to complications.

Table 1: Various outcomes of the affected women*+.

\begin{tabular}{|lll|}
\hline & Number & $\begin{array}{l}\text { Percentage } \\
\text { the total } \\
(\mathbf{n}=\mathbf{7 8})\end{array}$ \\
\hline Preeclampsia & $\mathbf{9}$ & $\mathbf{1 1 . 5 4} \%$ \\
\hline Early onset PE & 6 & $7.69 \%$ \\
\hline Late onset PE & 3 & $3.85 \%$ \\
\hline IUGR & $\mathbf{2 5}$ & $\mathbf{3 2 . 0 5} \%$ \\
\hline $\begin{array}{l}\text { IUGR with EO- } \\
\text { PE }\end{array}$ & 5 & $6.41 \%$ \\
\hline $\begin{array}{l}\text { IUGR without } \\
\text { PE }\end{array}$ & 20 & $25.64 \%$ \\
\hline Total & $\mathbf{2 9}$ & $\mathbf{3 7 . 1 8} \%$ \\
\hline
\end{tabular}

*There was one case of abruption placentae at 35 weeks of gestation in a woman with early onset preeclampsia and IUGR and one antepartum intrauterine death occurred in a woman with early onset severe pre-eclampsia with severe IUGR at 29 weeks 4 day of gestation; ${ }^{+} \mathrm{PE}$ - preeclampsia, EO-PE - early onset preeclampsia; IUGR- intrauterine growth restriction.

In present study, all women enrolled were of Indian origin, had a spontaneous conception, none of the women enrolled were APLA positive or had pre-existing diabetes 
mellitus or renal disease or had age more than 35 years or history of smoking. The mean age of the women who developed complications was 26.48 years and those who did not was 25.61 years $(\mathrm{p}=0.33)$. The mean BMI of women with complications was $24.36 \mathrm{~kg} / \mathrm{m}^{2}$ and of those without complications was $24.59 \mathrm{~kg} / \mathrm{m}^{2}$ ( $\mathrm{p}=0.803$ ). No statistically significant increase in complications was found in primigravida (relative risk $=0.65,95 \% \mathrm{CI}=$ $0.36-1.15, \mathrm{p}=0.2$ ). History of preeclampsia and related complications in previous pregnancy did not have a statistically significant impact on the outcome of the present pregnancy (relative risk $=1.54(95 \% \mathrm{CI}=0.588$ $3.098, \mathrm{p}=0.88$ ). Family history of hypertension or diabetes did not statistically affect the development of complications in pregnancy (relative risk $=1.54,95 \%$ $\mathrm{CI}=0.54-2.068, \mathrm{p}=0.915)$.

Table 2: Various outcomes of the affected women*+.

\begin{tabular}{|llll|}
\hline & $\begin{array}{l}\text { Women with } \\
\text { complications } \\
\mathrm{n}=29\end{array}$ & $\begin{array}{l}\text { Women } \\
\text { without } \\
\text { complications } \\
\mathrm{n}=49\end{array}$ & $\begin{array}{l}\text { Level of } \\
\text { significance }\end{array}$ \\
$\begin{array}{l}\text { Mean } \\
\text { MAP } \\
\pm \text { SD }\end{array}$ & $78.62 \pm 23.15$ & $81.62 \pm 8.55$ & $\mathrm{p}=0.415$ \\
$\mathrm{l}=0.819$
\end{tabular}

*PE - preeclampsia; MAP - mean arterial pressure; PAPP-A pregnancy associated plasma protein - A; PI - pulsatility index; SD - standard deviation.

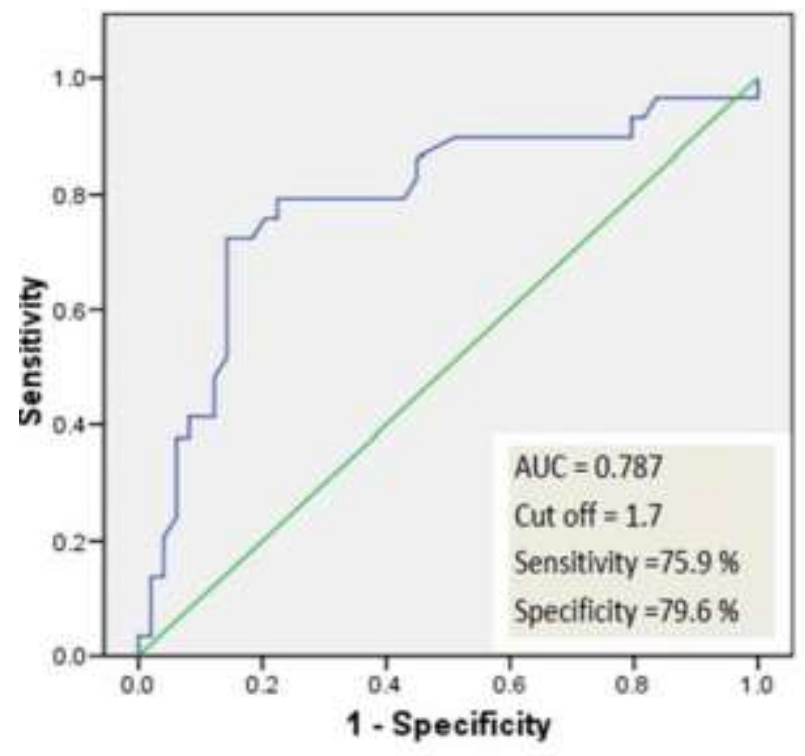

Diagonal segments are produced by ties

Figure 1: Receiver operator characteristic curve of mean PI.
Table 2 shows the mean MAP, mean PAPP-A and mean of mean PI in women with different complications in relation with women without complications. The mean MAP (mean arterial pressure) of the women who developed preeclampsia and related complications was $78.62( \pm 23.15)$ and that of women who did not develop complications was $81.62 \pm 8.55$. The mean PAPP-A of the women who developed preeclampsia and related complications was $14.20 \pm 11.05$ and that of women who did not develop complications were $13.89 \pm 7.13$. Both these comparisons were not statistically significant.

The mean of mean PI of the women who developed preeclampsia and related complications was $1.94 \pm 0.55$ and that of women who did not develop complications was $1.42 \pm 0.44$. The comparison of these was statistically significant ( $\mathrm{p}<0.001)$.

$19(24.36 \%)$ of the women enrolled had presence of an early diastolic notch on Uterine Artery Doppler at 11-14 weeks of pregnancy. 13 of these women developed complications and 6 did not develop complications. The relative risk of development of complications in women with presence of 'notching' was $2.48(95 \% \mathrm{CI}=1.479-$ $4.159, \mathrm{p}=0.004)$. This value was statistically significant. Of these women, 4 developed preeclampsia and 10 developed IUGR. The relative risk of development of preeclampsia in the presence of notch was 2.48 (95\% CI $=0.742$ to $8.322, \mathrm{p}=0.28)$ but this was not statistically significant. Risk of development of IUGR is 2.22 (95\% $\mathrm{CI}=1.187-4.146, \mathrm{p}=0.037)$ and this was statistically significant.

A Receiver Operating Characteristic curve (or ROC curve) was then plotted for mean PI and the following results were obtained (Figure 1).

The AUC (area under curve) of the mean PI ROC was $0.787(95 \% \mathrm{CI}=0.675-0.900)$ for a cut off of 1.7 . The sensitivity of mean PI at 11-14 weeks of pregnancy for predicting complications is $75.9 \%$ and specificity is 79.6 $\%$. Thus mean PI was found to be the best single investigation to predict preeclampsia and IUGR.

\section{DISCUSSION}

The possibility of prediction of preeclampsia in first trimester of pregnancy is a fairly new concept and although in recent years, studies combining various parameters at 11-14 weeks of pregnancy have been undertaken; an algorithm with a high predictive value is yet to be developed. Additionally, none of these studies have been done for Indian women.

The findings of this study demonstrate that the women who developed the above mentioned complications were demographically similar to women who did not develop complications. Various studies have found that women with antiphospholipid antibodies, a history of preeclampsia, pre-existing diabetes, multiple pregnancy, 
family history, nulliparity, a raised BMI before pregnancy or at booking, maternal age $>40$, renal disease and hypertension all increased the risk of a woman developing preeclampsia. ${ }^{19}$ These results were not reciprocated in this study because only a limited number of women were enrolled. Additionally they were recruited from the general population and did not have pre-existing high risk factors like chronic hypertension and multifetal pregnancy.

A nested case control study done in Netherlands by Kuc et al found first-trimester MAP to be one of the most important predictors of PE. ${ }^{7}$ In case of EO-PE, MAP was significantly higher (cut-off - 1.04 MAP MoM, p = 0.0001 ) compared to controls. Also higher MAP MoM were found in LO-PE pregnancies (MAP - 1.05 MoM, $\mathrm{p}=$ 0.0001).MAP was again found to be statistically significantly increased in the EO-PE and IUGR group as compared to controls $(1.04 \mathrm{MoM}, \mathrm{p}=0.01)$ but not for IUGR alone. In present study, we did not find a corelation between development of preeclampsia and IUGR to MAP.

Although a low PAPP-A in itself is not a strong indicator of preeclampsia; studies have previously shown a significant improvement in detection by combining first trimester PAPP-A measurement with uterine artery Doppler velocimetry. ${ }^{7,20}$ In present study, PAPP-A was not found to be predictive of preeclampsia and related complications.

A recent meta-analysis by Velauthar et al, reviewed the accuracy of uterine artery Doppler analysis in the first trimester in the prediction of IUGR and preeclampsia. ${ }^{21}$ Eighteen studies involving 55974 women were evaluated, with fifteen of these studies enrolling women with low risk pregnancies. Uterine artery RI or PI $\geq 90$ th centile and the presence of notching (unilateral/bilateral) were used to define abnormal flow velocity waveforms. An abnormal uterine artery PI in the first trimester was predictive of preeclampsia and early-onset preeclampsia with sensitivities of $26.4 \%$ and $47.8 \%$, respectively. Fetal growth restriction was predicted at $15.4 \%$.

In present study, uterine artery PI at 11-14 weeks of pregnancy was found to be the best parameter for screening of women at risk of developing preeclampsia and IUGR as it had a high sensitivity (75.9\%) and specificity $(79.2 \%)$ for identifying the high risk group at a cut of 1.70 .

Thus uterine artery Doppler alone is a good screening test for prediction of preeclampsia and related complications in the first trimester, especially in a developing nation like India where there are limited resources.

This study has been conducted in a limited number of pregnant women and results cannot be extrapolated to a larger population. Nevertheless, the trends of uterine artery Doppler PI have been similar to international studies. A larger study is needed in an Indian scenario to validate the findings of this study.

Funding:ICMR MD thesis grant

Conflict of interest: None declared

Ethical approval: The study was approved by the Institutional Ethics Committee (ref. code 70th ECM IIB/P47)

\section{REFERENCES}

1. Cunningham FG, Lenovo KJ, Bloom SL, Spong KY, Dashe JS, Hoffman BL, Casey BM, Sheffield JS. Williams Obstetrics. Twenty-Fourth Edition. United States of America: McGraw-Hill Education; 2014.

2. Bilano VL, Ota E, Ganchimeg T, Mori R, Souza JP. Risk Factors of Pre-Eclampsia/Eclampsia and Its Adverse Outcomes in Low- and Middle-Income Countries: A WHO Secondary Analysis. Young RC, ed. PLoS ONE. 2014;9(3):e91198.

3. Say L, Chou D, Gemmill A, Global causes of maternal death: a WHO systematic analysis. Lancet Glob Health. 2014 2:e323-33

4. Konar H, Chakraborty AB, Maternal mortality: A FOGSI study (Based on institutional data). J Obstet Gynaecol India. 2013;63(2):88-95.

5. Montgomery AL, Ram U, Kumar R, Jha P, for The Million Death Study Collaborators. Maternal Mortality in India: Causes and Healthcare Service Use Based on a Nationally Representative Survey. Bhutta ZA, ed. PLoS ONE. 2014;9(1):e83331.

6. Khong SL, Kane SC, Brennecke SP, da Silva Costa F. First-Trimester Uterine Artery Doppler Analysis in the Prediction of Later Pregnancy Complications. Disease Markers. Volume 2015, Article ID 679730

7. Kuc S, Koster MPH, Franx A, Schielen PCJI, Visser GHA. Maternal Characteristics Mean Arterial Pressure and Serum Markers in Early Prediction of Preeclampsia. PLoS ONE. 2013;8(5):e63546.

8. Pilalis A, Souka AP, Antsaklis P, Daskalakis G, Papantoniou N, Mesogitis S. Screening for preeclampsia and fetal growth restriction by uterine artery Doppler and PAPP-A at 11-14 weeks' gestation.Ultrasound Obstet Gynecol. 2007;29:13540

9. Zhong Y, Tuuli M, OdiboAO. First-trimester assessment of placenta function and the prediction of preeclampsia and intrauterine growth restriction. Prenatal Diagnosis. 2010;30(4):293-308.

10. Huppertz B. Placental origins of preeclampsia: challenging the current hypothesis.Hypertension. 2008;51(4):970-5.

11. Fleiss JL, Levin B, Paik MC. Statistical methods for rates and proportions. Third Edition. Wiley. 2003.

12. Noninvasive prenatal testing for fetal aneuploidy. Committee Opinion No.545, American College of Obstetrics and Gynecologists. Obstet Gynecol. 2012;120:1532-4.

13. Akolekar R, Syngelaki A, Sarquis R, Zvanca M, Nicolaides KH. Prediction of early, intermediate and 
late pre-eclampsia from maternal factors, biophysical and biochemical markers at 11-13 weeks. Prenat Diagn. 2011;31:66-74.

14. Poon LCY, Kametas NA, Pandeva I, Valencia C, Nicolaides KH. Mean Arterial Pressure at $11+0$ to $13+6$ Weeks in the Prediction of Preeclampsia. Hypertension. 2008;51:1027-33.

15. ISUOG Practice Guidelines: use of Doppler ultrasonography in obstetrics. Ultrasound Obstet Gynecol. 2013;41:233-9.

16. American College of Obstetricians and Gynecologists: Hypertension in pregnancy.Report of the American College of Obstetricians and Gynecologists'Task Force on Hypertension in pregnancy. Obstet Gynecol. 2013;122:1122.

17. Mikolajczyk RT, Zhang J, Betran AP, Souza JP, Mori R, Gülmezoglu AM, et al. A global reference for fetal-weight and birthweight percentiles. Lancet. 2011;377:1855-61.

18. Hadlock FP, Harrist RB, Martinez-Poyer J. In utero analysis of fetal growth: a sonographic weight standard. Radiology. 1991;181:129-33.

19. Duckitt K, Harrington D. Risk factors for preeclampsia at antenatal booking: systematic review of controlled studies. BMJ. 2005;330:565-72.

20. Spencer K, Cowan NJ, Nicolaides KH .Low levels of maternal serum PAPP-A in the first trimester and the risk of pre-eclampsia. Prenat Diagn. 2008;28:7-10.

21. Velauthar L, Plana ML, Kalidindi M. First-trimester uterine artery Doppler and adverse pregnancy outcome: a metaanalysis involving 55974 women. Ultrasound in Obstetrics and Gynecology. 2014;43(5):500-7.

Cite this article as: Narang S, Agarwal A, Das V, Pandey A, Agrawal S, Ali W. Prediction of preeclampsia at 11-14 weeks of pregnancy using mean arterial pressure, uterine artery Doppler and pregnancy-associated plasma protein-A. Int J Reprod Contracept Obstet Gynecol 2016;5:3948-53. 